\title{
e-Migrinter
}

$14 \mid 2016$

L'ajustement méthodologique comme fabrique critique du savoir dans les études migratoires

\section{L'ajustement méthodologique comme fabrique critique du savoir dans les études migratoires}

Mike Gadras et Josepha Milazzo

\section{OpenEdition}

\section{Journals}

Édition électronique

URL : https://journals.openedition.org/e-migrinter/686

DOI : 10.4000/e-migrinter.686

ISSN : 1961-9685

Éditeur

UMR 7301 - Migrinter

Référence électronique

Mike Gadras et Josepha Milazzo, «L'ajustement méthodologique comme fabrique critique du savoir dans les études migratoires », e-Migrinter [En ligne], 14 | 2016, mis en ligne le, consulté le 20 mai 2021. URL : http://journals.openedition.org/e-migrinter/686 ; DOI : https://doi.org/10.4000/e-migrinter.686

Ce document a été généré automatiquement le 20 mai 2021.

Tous droits réservés 


\title{
L'ajustement méthodologique comme fabrique critique du savoir dans les études migratoires
}

\author{
Mike Gadras et Josepha Milazzo
}

Nous souhaiterions remercier les auteurs pour leur investissement dans la réalisation de ce dossier thématique. Nous adressons également notre vive reconnaissance à Cédric Audebert, directeur de la publication e-Migrinter, pour nous avoir donné la possibilité de réaliser ce projet, ainsi qu'à toute l'équipe du secrétariat de rédaction de la revue et aux relecteurs scientifiques.

1 Ce numéro de la revue e-Migrinter vient concrétiser le projet de rassembler une partie des communications orales présentées lors d'un séminaire tenu à la Maison des Sciences de l'Homme et de la Société de l'Université de Poitiers, les 23 et 24 juin 2014, et organisé par les doctorants du laboratoire MIGRINTER: "Nouvelles migrations, nouveaux concepts, nouvelles méthodes. Vers une réflexion critique sur l'enthousiasme pour la nouveauté dans le champ des migrations». À l'origine de ce séminaire, se trouvait la volonté d'apporter des éléments de réponse quant à la réflexivité sur les méthodes de production des données dans les études migratoires ${ }^{1}$. C'est dans ce cadre et dans cette perspective qu'a été créé le Réseau Migrations ${ }^{2}$, qui constitue aujourd'hui un espace dynamique d'échanges et de synergies pour les doctorants et les jeunes chercheurs intéressés par les études migratoires. Ce numéro d'e-Migrinter constitue la première publication rassemblant de jeunes chercheurs, doctorants et docteurs, ainsi membres du « Réseau ».

2 Ces deux journées de séminaire ${ }^{3}$ avaient pour enjeu de questionner l'efficience de méthodes mixtes/combinées/transdisciplinaires de recherche et d'outils théoriques, dont nous nous servons pour appréhender et comprendre les phénomènes migratoires sur lesquels portent nos recherches doctorales. Compte tenu des évolutions des travaux des chercheurs, il nous a semblé opportun de faire du processus itératif - qu'est la construction du savoir scientifique - le fil d'Ariane de ce dossier. Le numéro est à ce titre organisé en quatre axes: (1) "visibilisation» de sources inédites et réinterprétations de phénomènes migratoires, (2) problèmes méthodologiques de 
l'étude de populations dispersées et mobiles, (3) adaptabilité d'approches inhabituelles aux études migratoires, et (4) opérationnalité de notions et de concepts redéfinis au prisme de la migration. Pour ce numéro d'e-Migrinter, les différentes contributions de ses auteurs vous invitent ainsi à explorer la problématique de l'ajustement méthodologique comme fabrique critique du savoir dans les études migratoires, selon différentes facettes.

3 Pour illustrer ces sujets de réflexion, nous vous proposons un recueil de huit textes, soit cinq articles classiques et trois articles-résumés de posters scientifiques ${ }^{4}$. Les contributions réunies interrogent selon différentes perspectives, les relations entre les apports théoriques, les démarches méthodologiques, les méthodes et les sources de données utilisées. Ces études s'inscrivent dans des disciplines ${ }^{5}$, s'insèrent dans des champs thématiques et s'appuient sur des aires géographiques d'une grande diversité. Usant de réflexivité à des stades d'avancement distincts, les auteurs s'attachent à justifier la pertinence de leurs choix : ils interrogent l'opérationnalité de ceux-ci, selon les questionnements et les réajustements consécutifs ayant ponctué leurs recherches. Ces textes nous permettent ainsi, un à un, de distinguer quelles ont été les sinuosités du cheminement particulier à chacun, au fil de la construction de la recherche. Ce dialogue entre théorie et empirie permet de comprendre les mécanismes de chacune et in fine leurs liens dynamiques (Ruby, 2013, pp.1009-1010). Et au regard de ce "dynamisme » et de ces "bricolages méthodologiques » inhérents à la création du savoir, il convient de souligner la flexibilité et l'adaptabilité dont le chercheur doit faire preuve, notamment dans les études migratoires (Castles, 2012, p. 16).

4 La première partie du dossier porte sur l'importance du choix des sources dans la connaissance du phénomène migratoire étudié. Deux articles soulignent l'incidence de l'utilisation de sources jusqu'alors inexploitées. Valentin Guillaume interroge le quotidien ordinaire des " petits " réfugiés politiques polonais présents dans les villages de l'ouest de la France du XIX ${ }^{\text {ème }}$ siècle. Cette population constituait jusqu'alors « une majorité silencieuse et méconnue» de l'émigration polonaise. L'historiographie européenne avait en effet fait des élites polonaises au sein de la capitale française les seuls "représentants» de cette émigration. L'auteur apporte ainsi une vision plus complexe de la "Grande Émigration polonaise", et subséquemment, de nouveaux éléments sur la migration politique et les conditions de l'exil. Laure Sizaire s'intéresse aux migrations par le mariage, un champ de recherche peu étudié et parent pauvre de la littérature sociologique francophone. La prétendue nouveauté de ces migrations dans la littérature - qu'interroge précisément l'auteure - révèle que certaines femmes migrantes par le mariage restent "dans l'ombre». À partir de contextes nationaux postsoviétiques, l'auteure offre un regard particulier sur les logiques qui sous-tendent ces projets matrimoniaux et migratoires. Elle s'écarte des discours binaires dominants portés sur ces femmes, oscillant entre « la victime» et «la manipulatrice», et définit ces parcours migratoires tels des « carrières » matrimoniales et mobiles, témoignant de l'adaptabilité et de la capacitation de ces femmes « actrices ».

5 La deuxième partie interroge certains problèmes méthodologiques posés par l'étude de populations migrantes dispersées et mobiles. Deux articles illustrent la combinaison de méthodologies et de méthodes de recherche pour répondre aux enjeux de l'approche multi-sites. Pour répondre au défi d'ethnographies multi-sites synchrones et sur le temps long, Ilka Vari-Lavoisier revient sur l'élaboration d'un protocole d'enquête transnational, interdisciplinaire et collaboratif. L'enjeu est double. Il s'agit d'une part 
de restituer les stratégies migratoires. D'autre part, elle interroge le rôle occupé par les absents dans les différents espaces liés par des pratiques et des activités de migrants. En mutualisant le recueil et l'analyse de données qualitatives, l'équipe a ainsi pu saisir les dimensions économiques et sociales des transferts d'argent de migrants sénégalais vivant en France, depuis le Sénégal. La dimension collective du projet a servi à un pluralisme des points de vue locaux des enquêteurs et à une réflexivité sur les modalités de recherche au sein de l'équipe. Kelly Poulet se propose d'analyser la migration au prisme de son absence de mouvement géographique, selon des temporalités et des spatialités mobilitaires restreintes et en stand-by, à défaut d'être effectives. Partie au départ pour suivre les circulations de jeunes dakarois entre Paris et Dakar (Sénégal), elle s'interroge finalement sur la manière de saisir les trajectoires migratoires de ceux, majoritaires, qui ne partent pas. Elle explicite sa pratique spécifique du terrain multi-situé : le "nomadisme sédentaire » lui permet de rendre compte de la reconstruction des projets et des espoirs des migrants, selon les contraintes d'une mobilité assignée à l'immobilité. L'auteure livre ainsi une réflexion sur les rapports entre logiques de sédentarité et de mobilité, et sur la part de chacune qui réside « en puissance » dans l'autre.

6 La troisième partie aborde l'adaptabilité de méthodologies inhabituelles au champ des études migratoires. Deux articles prennent part à de possibles réflexions sur l'intérêt de telles démarches pour étudier la migration. Selon «l'approche situationnelle », David Puaud soulève la question de la pertinence de ce cadre opératoire appliqué à l'étude de phénomènes migratoires. Son étude porte ici sur l'analyse, lors d'un procès d'assises, des enjeux symboliques et identitaires de l'explication d'un crime par la seule biographie psychosociale d'un individu et sa description comme "monstre humain ", selon les accusations à sa charge. L'auteur montre comment ces enjeux peuvent entrer en conflit avec une compréhension des faits qui reposerait à la différence sur l'analyse des contextes, des situations et des trajectoires sociales. Travailleur social et chercheur en anthropologie, l'approche situationnelle favoriserait à cet égard des modalités de collecte de données qui optimisent en retour l'enquête scientifique. Elle permet au chercheur d'adopter un regard distancié face à son objet de recherche, autrement dit ici : analyser la subtilité des interactions humaines en jeu, «les séquences » liées aux différentes audiences et les enjeux symboliques liés au dispositif du procès. Appliquée à l'étude de populations migrantes, on pourrait à titre d'exemple évoquer l'intérêt de cette approche pour contourner la catégorisation essentialiste réduisant les personnes à leur seul statut de "migrants ». Mike Gadras entend saisir comment s'élabore l'expérience migratoire, en s'appuyant sur la recherche biographique, la philosophie et l'anthropologie. Il propose une méthodologie novatrice, distincte des approches biographiques traditionnelles: il combine des problématiques liées au mode de compréhension de l'expérience, à des enjeux attenant à l'interprétation herméneutique. En s'appuyant sur l'examen d'entretiens menés auprès de migrants en situation "irrégulière ", il invite à appréhender leur "parole vive ", soit : ce qu'un individu dit de son vécu, les significations qu'il confère à son expérience propre, et comment cette expérience est mise en forme par les procédés narratifs qu'utilisent ces personnes.

7 La quatrième partie s'intéresse à la redéfinition de notions et de concepts au gré de phénomènes migratoires observés. Un article vient éclairer plus particulièrement les multiples allers retours empirie-théorie et la combinaison de questions tant descriptives qu'analytiques, constitutives de la recherche. Morgane Dujmovic discute la 
notion de frontière, qu'elle interroge au regard des modalités du contrôle migratoire dans un contexte particulier : celui, politico-territorial et géographique, de la Croatie située aux marges de l'Union européenne. L'auteure considère la frontière telle un objet géographique multidimensionnel et multi-scalaire. Subséquemment, elle montre comment la mise en place d'une méthodologie faite de «bricolages " vient répondre à l'adaptabilité dont le chercheur doit user pour saisir les différents jeux d'échelles et d'acteurs dans le cadre de son sujet. Josepha Milazzo discute l'opérationnalité du concept de village global, à la lumière des transformations de la commune villageoise espagnole de Cadaqués dans la mondialisation. Elle propose un regard distancié à l'égard des paradigmes urbains dominants qui font des villes globales et des métropoles les points de référence analytiques de la mondialisation et de la migration. Interroger les rapports entre l'échelle, les formes d'incorporation des migrants et les transformations de l'espace, permet de comprendre comment ce village a évolué et évolue. L'objectif est ainsi de montrer le rôle que peuvent jouer les espaces villageois dans la compréhension de la mondialisation.

Force est de constater que l'ensemble des articles donne à voir un souci similaire de distanciation vis-à-vis des cadres épistémologiques, théoriques et/ou méthodologiques choisis, pour mieux les confronter, les tester et les "réajuster». L'ajustement méthodologique dans les études migratoires, questionné tel un ensemble de procédés dont la pertinence doit être passée au crible de la critique (DeWind et Holdaway, 2007), se révèle ainsi un sujet de réflexion à part entière. Il nous éclaire sur le sens que le chercheur peut donner aux informations recueillies et sur les contextes de production $\mathrm{du}$ savoir. Pour refermer le propos, nous pouvons à ce titre remarquer que «la méthodologie est notre carte pour naviguer dans le monde social, tandis que les méthodes constituent les outils de notre entreprise» (Castles, 2012, p. 7, traduction libre des auteurs).

\section{BIBLIOGRAPHIE}

Castles, Stephen (2012) Understanding the relationship between methodology and methods, in Vargas-Silva, C. (Éd.) Handbook of Reaseach Methods in Migration, Cheltenham, Northampton, Edward Elgar Publishing Limited, pp. 7-25.

DeWind, Josh ; Holdaway, Jennifer (2007) Preface, in DeSipio, L. ; Garcia y Griego, M. ; Kossoudji, S. (Éds.) Researching Migration: Stories from the Field, New York, Social Science Research Council, pp. 9-12, [en ligne, réf. du 15 février 2016]. [Disponible sur Internet].

Ruby, Christian (2013) Théorie / Empirie, in Lévy, J. ; Lussault, M. (Éds.) Dictionnaire de la géographie et de l'espace des sociétés, Paris, Belin, pp. 1009-1010. 


\section{NOTES}

1. La réflexivité sur les méthodes de production de données dans le champ d'étude des migrations internationales est une préoccupation de recherche dont on peut à ce titre prendre la mesure avec le cycle de séminaires « Sources et Méthodes » du laboratoire Migrinter.

2. Le Réseau Migrations compte désormais une centaine de membres et entend rassembler les jeunes chercheur.e.s dont les travaux placent les questions migratoires au cœur de leurs préoccupations. Il a vocation à leur offrir un espace interdisciplinaire, professionnel comme informel, d'échanges, d'entraides et de réflexions. À ces desseins, le réseau organise divers événements scientifiques de même qu'il dispose d'une plateforme numérique de partage des activités et des travaux intéressés aux études migratoires [en ligne réf. du 15 février 2016].

3. Un compte-rendu détaillé de ce séminaire est disponible sur la page internet hypothèses.org du laboratoire Migrinter [en ligne, réf. du 15 février 2016].

4. Lors de ce séminaire, dix communications classiques et cinq communications sous le format "poster scientifique » avaient alors été présentées.

5. Les articles rassemblés embrassent des disciplines aussi variées que celles de la géographie, des sciences de l'éducation, de l'histoire, de la sociologie, de l'anthropologie et des sciences sociales en civilisation.

\section{AUTEURS}

\section{MIKE GADRAS}

Doctorant en Sciences de l'Éducation, Paris 13 Sorbonne Paris Cité (EXPERICE)

mike.gadras@gmail.com

JOSEPHA MILAZZO

Doctorante en Géographie, Aix-Marseille Université (TELEMMe) / Universitat Autònoma de Barcelona (Departamento de Geografía)

jjomilazzo@gmail.com 